Case Report

\title{
Severe Hypercholesterolemia Associated with Steroid-Refractory Acute Liver GVHD: Unusual Complication of Allogeneic Hematopoietic Cell Transplantation
}

Bhagyashree Deshmankar ${ }^{1}$, Craig E. Eckfeldt ${ }^{1}$, Graf Oliver ${ }^{2}$, Aleksandr Lazaryan ${ }^{3}$, Nelli Bejanyan ${ }^{3}$,

1. Hematology, Oncology and Transplantation, University of Minnesota, Minneapolis, MN, USA; EMails: Drbhagyashree.deshmankar@gmail.com; eckf0002@umn.edu

2. Surgical Pathology, University of Minnesota, Minneapolis, MN, USA; E-Mail: graf0151@umn.edu

3. Blood and Marrow Transplant and Cellular Immunotherapy, Moffitt Cancer Center, Tampa, FL, USA; E-Mails: aleksandr.lazaryan@moffitt.org; nelli.bejanyan@moffitt.org

* Correspondence: Nelli Bejanyan; E-Mail: nelli.bejanyan@moffitt.org

Academic Editor: Yvonne A. Efebera

Special Issue: Graft-versus-Host Disease

OBM Transplantation

2020, volume 4, issue 3

doi:10.21926/obm.transplant.2003118
Received: July 19, 2020

Accepted: September 04, 2020

Published: September 18, 2020

\begin{abstract}
Severe hypercholesterolemia is an uncommon complication of allogenic hematopoietic cell transplantation (HCT). Treatment options for hypercholesterolemia due to liver graft-versushost disease (GVHD) are limited because many antilipidemic medications are known to worsen hepatic functions. We report our experience on a case of a 38-year-old male with Philadelphia chromosome positive acute lymphoblastic leukemia presenting with steroidrefractory hepatic and lower gastrointestinal acute GVHD after matched sibling donor peripheral blood myeloablative HCT who developed severe hypercholesterolemia $(>1,000 \mathrm{mg} / \mathrm{dl})$. The patient was effectively treated with extracorporeal photopheresis for GVHD and severe hypercholesterolemia resolved after weeks of therapy with lipoprotein apheresis.
\end{abstract}

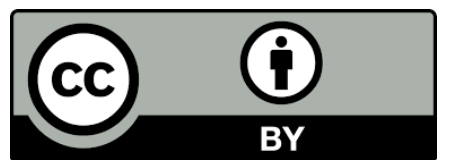

(C) 2020 by the author. This is an open access article distributed under the conditions of the Creative Commons by Attribution License, which permits unrestricted use, distribution, and reproduction in any medium or format, provided the original work is correctly cited. 


\section{Keywords}

Acute GVHD; lipid apheresis; severe hypercholesterolemia

\section{Introduction}

Severe hypercholesterolemia $(>1,000 \mathrm{mg} / \mathrm{dl}$ ) associated with graft-versus-host disease (GVHD) of the liver is a rare complication of allogenic hematopoietic cell transplantation (HCT) [1]. The etiology of hypercholesterolemia after HCT can be multifactorial. Several medications used for GVHD prophylaxis or treatment after HCT are known to cause dyslipidemia, including cyclosporine, tacrolimus, sirolimus and glucocorticoids [2-6]. However, the elevation of total cholesterol from these medications usually ranges between 200 to $300 \mathrm{mg} / \mathrm{dl}$ [7]. Severe hypercholesterolemia due to medications alone is rare. Treatment options for hypercholesterolemia due to hepatic GVHD are limited because many antilipidemic medications can further worsen liver function. In patients with severe hypercholesterolemia unresponsive to anti-hypercholesterolemic medications lipoprotein apheresis is a potentially effective treatment to prevent acute complications such as stroke, myocardial infarction, acute pancreatitis or hyperviscocity syndrome. Here, we present a case of a 38-year-old male with Philadelphia chromosome positive acute lymphoblastic leukemia (ALL) presenting with steroid-refractory acute GVHD of the liver and lower gastrointestinal tract after myeloablative matched sibling donor peripheral blood HCT who developed severe hyperlipidemia requiring lipoprotein apheresis. The patient provided written informed consent prior to proceeding with HCT.

\section{Case Description}

A 38-year-old male presented with high-grade fever, left ear pain, fatigue, and lightheadedness. He was found to have hyperleukocytosis with a white blood cell count of $380,000 / \mathrm{mL}$ and $85 \%$ circulating peripheral blasts, associated with anemia and thrombocytopenia. Bone marrow biopsy was performed showing $72 \%$ lymphoblasts by morphology and $87 \%$ immunophenotypically abnormal cells (CD34+, CD43+, CD19+, CD10+, CD13+, CD33+, CD79at, TdT+ and HLA-DR+) suggestive of B-cell ALL. Cytogenetic analysis demonstrated translocation $(9 ; 22)$ and loss of chromosome 7. Additionally, BCR-ABL molecular testing by polymerase chain reaction (PCR) was positive for p210 transcript. The patient received chemotherapy with hyper-CVAD (cycles $1 A-B$ ) in combination with imatinib [8]. Diagnostic lumbar puncture (LP) showed no evidence of leukemia in cerebrospinal fluid (CSF) by flow cytometry or cytomorphology testing. He received prophylactic intrathecal chemotherapy with methotrexate and cytarabine based on the hyper-CVAD protocol [8]. Bone marrow biopsy performed after cycle 1B of hyper-CVAD chemotherapy revealed a $70-80 \%$ cellular marrow with no morphologic evidence of B-ALL. However, flow cytometry showed $1 \%$ residual lymphoblasts and molecular testing revealed a BCR-ABL1/ABL1 ratio of $3.4 \%$. Tyrosine kinase inhibitor imatinib was replaced by dasatinib, while he continued with subsequent cycles of hyper-CVAD (2A-3A) that led to gradual reduction in his measurable residual disease (MRD) level. Subsequently, morphological and immunophenotypic complete remission was achieved. However, no further reduction of MRD could be achieved molecularly and he underwent a myeloablative 
matched sibling donor peripheral blood HCT with cyclophosphamide (120mg/kg total dose) and total body irradiation (1,320 cGy) conditioning. At the time of HCT he had a detectable molecular MRD by PCT testing (BCR-ABL1/ABL1=0.58\%). GVHD prophylaxis consisted of cyclosporine and methotrexate (MTX).

At day +31 after transplant, the patient presented with diarrhea and elevated liver function tests (LFTs). Flexible sigmoidoscopy with low GI biopsy confirmed histological evidence of acute GVHD, which was of clinical grade III acute GVHD (stage 3 gastrointestinal and stage 2 liver) [9]. Despite treatment with high-dose methylprednisolone he had progressively worsening GI symptoms (abdominal pain and hematochezia) and LFT abnormalities, suggestive of steroid-refractory acute GVHD. The patient received bi-weekly subcutaneous etanercept (25 $\mathrm{mg} \times 8$ doses) with initial achievement of partial response in all GVHD target organs [10]. However, following the completion of etanercept therapy his LFT abnormalities progressivity worsened with total bilirubin of 17.7 $\mathrm{mg} / \mathrm{dL}$, ALT of $863 \mathrm{U} / \mathrm{L}$, AST of $365 \mathrm{U} / \mathrm{L}$ and alkaline phosphatase of $325 \mathrm{U} / \mathrm{L}$. Dasatinib was discontinued and liver ultrasound with vascular doppler showed no evidence of sinusoidal obstruction syndrome (SOS) or any hepatic pathology. Liver biopsy was performed and it demonstrated cholestasis, bile duct damage, and ductopenia suggestive of hepatic acute GVHD (Figure 1).

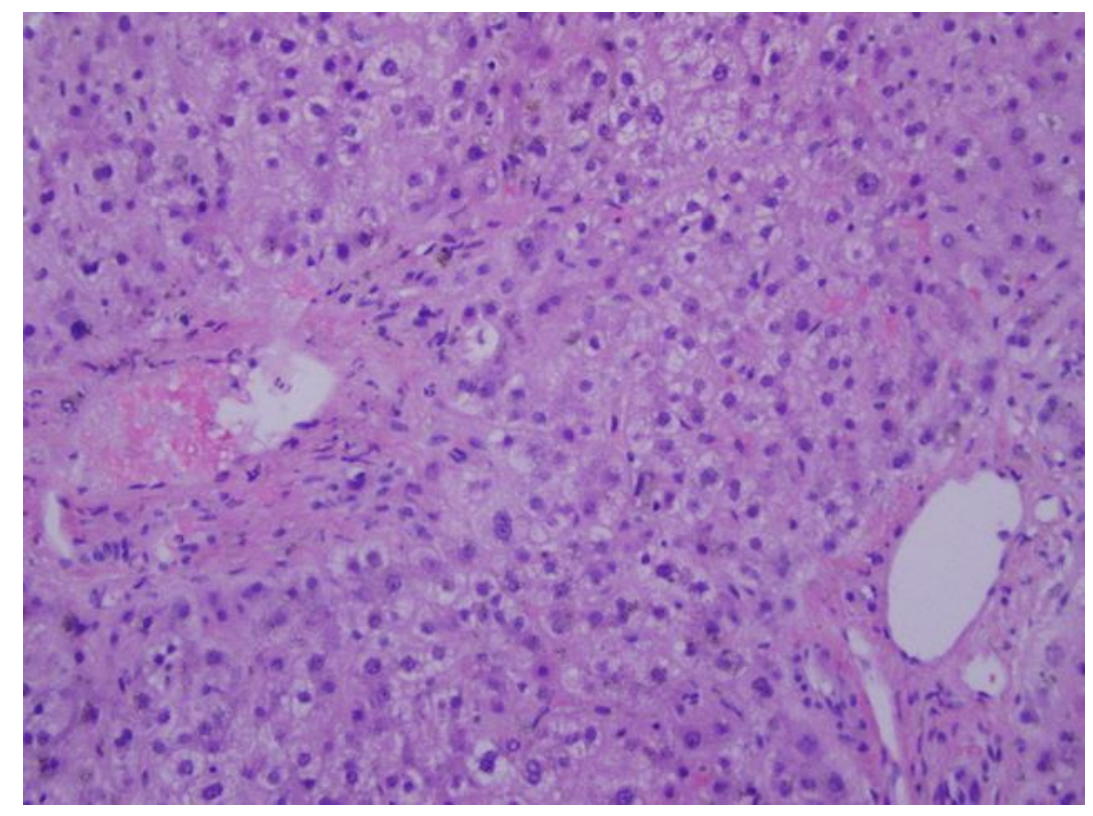

Figure 1 Liver biopsy of the patient with acute hepatic GVHD.

The patient received horse antithymocyte globulin (ATG; $30 \mathrm{mg} / \mathrm{kg} /$ day) from days +51 to +56 of HCT with no response [10]. In addition, bi-weekly extracorporeal photopheresis (ECP) was initiated and continued until day +208 post-HCT, which led to gradual improvement and subsequent resolution of his GI symptoms and LFT abnormalities [11]. Dasatinib was resumed at day +100 after HCT with restaging bone marrow biopsy showing complete morphological and immunophenotypic remission but detectable molecular MRD. At day +180 post-HCT the patient was found to have hyponatremia (serum $\mathrm{Na}=124 \mathrm{mmol} / \mathrm{L}$ ), normal thyroid function tests, serum hyperviscosity (above detection limits) and severe hypercholesterolemia with non-fasting lipid panel showing direct lowdensity lipoprotein ( $L D L$ ) of $1,735 \mathrm{mg} / \mathrm{dL}$, triglycerides of $707 \mathrm{mg} / \mathrm{dL}$, and total cholesterol/ high- 
density lipoprotein (HDL) ratio of 91. The patient had no prior history of cardiovascular disease or family history of hypercholesterolemia. Persistent LFT abnormalities in a setting of hepatic GVHD indicated that antihyperlipidemic medications would be of limited use. Therefore, under direction of preventative cardiologist with expertise in lipid disorders, urgent lipoprotein apheresis was initiated to achieve fast reduction of blood lipid levels and to prevent acute pancreatitis or cardiovascular complications [12]. He required a total of 13 biweekly cycles of lipoprotein apheresis with effective reduction in blood lipid levels and subsequent resolution of severe hypercholesterolemia after achievement of hepatic GVHD complete response with ECP therapy.

Close monitoring of cholesterol levels is important in patients after HCT in order to avoid hypercholesterolemia related complications. In addition, hyponatremia in some patients can be the sole sign of hypercholesterolemia in absence of any clinical symptoms. Moreover, post-transplant immunosuppression or maintenance TKI therapy should be carefully chosen in patients with hepatic GVHD given the hyperlipidemic potential of certain immunosuppressive agents. For example, high incidence of hypercholesterolemia is reported in patients treated with nilotinib, thus either dasatinib or imatinib use is preferred as a maintenance TKI therapy in patients after HCT presenting with hypercholesterolemia [13]. In patients with mild to moderate hypercholesterolemia after allogeneic HCT, we recommend an initial medical management with use of antihyperlipidemic medications. Although statins are the most effective drugs to control LDL predominant hyperlipidemia [14], they frequently can result in elevations of hepatic transaminases and myopathy [15]. Most statins are dependent on hepatic cytochrome P-450 enzyme for metabolism and elimination. Thus, due to their toxicity profile and need for very close monitoring combination therapies with statins and strong 3A4 inhibitors are not desirable in allogeneic HCT recipients who frequently receive azoles or other 3A4 inhibitors [16]. In addition, statins are substrates of hepatic transporter proteins including organic anion transporting polypeptide, which can result in undesirable drug-drug interactions and further limit the use of statins in some patients [16]. Other frequently used anti-hypercholesterolemic medications include fibrates that can help reducing the triglycerides by 30-60\% [17]. N-3 polyunsaturated acids (N-3 PUFAs or "fish oils"), including docosahexaenoic acid and eicosapenaenoic acid, like omega-3-acid ethyl esters (Lovaza) are another group of effective agents that can be used to lower triglycerides [17]. These medications can be particularly useful in a setting of sirolimus-induced dyslipidemia or lipoprotein-X mediated hypercholesterolemia, which are characteristically triglyceride-predominant conditions [17]. Other anti-hypercholesterolemic medications include niacin, bile acid sequestrants, and ezetimibe [17]. However, these agents have not been systematically evaluated in the allogeneic HCT population.

Acute and severe rise in cholesterol levels in our patient with acute hepatic GVHD prompted urgent use of lipoprotein apheresis to avoid acute complications of severe hypercholesterolemia. Thus, in patients with severe hypercholesterolemia $(>1,000 \mathrm{mg} / \mathrm{dl})$ requiring aggressive lipid control and in those unresponsive to anti-hypercholesterolemic medications lipoprotein apheresis can be considered in order to avoid serious cardiovascular complications.

\section{Author Contributions}

Nelli Bejanyan: Conception of the project and critical revision; Bhagyashree Deshamankar: drafting the manuscript; Craig E. Eckfeldt: critical revision of article; Graf Oliver: critical revision of article; Aleksandr Lazaryan: critical revision of article; All authors approved the final version of the manuscript. 


\section{Competing Interests}

The authors have declared that no competing interests exist.

\section{References}

1. Vantyghem MC, Cornillon J, Decanter C, Defrance F, Karrouz W, Leroy C, et al. Management of endocrino-metabolic dysfunctions after allogeneic hematopoietic stem cell transplantation. Orphanet J Rare Dis. 2014; 9: 162.

2. Sholter DE, Armstrong PW. Adverse effects of corticosteroids on the cardiovascular system. Can J Cardiol. 2000; 16: 505-511.

3. Kuster GM, Drexel H, Bleisch JA, Rentsch K, Pei P, Binswanger U, et al. Relation of cyclosporine blood levels to adverse effects on lipoproteins. Transplantation. 1994; 57: 1479-1483.

4. Satterthwaite R, Aswad S, Sunga V, Shidban H, Bogaard T, Asai P, et al. Incidence of new-onset hypercholesterolemia in renal transplant patients treated with FK506 or cyclosporine. Transplantation. 1998; 65: 446-449.

5. Chueh SC, Kahan BD. Dyslipidemia in renal transplant recipients treated with a sirolimus and cyclosporine-based immunosuppressive regimen: Incidence, risk factors, progression, and prognosis. Transplantation. 2003; 76: 375-382.

6. Bejanyan N, Rogosheske J, DeFor TE, Lazaryan A, Arora M, Holtan SG, et al. Sirolimus and mycophenolate mofetil as calcineurin inhibitor-free graft-versus-host disease prophylaxis for reduced-intensity conditioning umbilical cord blood transplantation. Biol Blood Marrow Transplant. 2016; 22: 2025-2030.

7. Turchin A, Wiebe DA, Seely EW, Graham T, Longo W, Soiffer R. Severe hypercholesterolemia mediated by lipoprotein $\mathrm{X}$ in patients with chronic graft-versus-host disease of the liver. Bone Marrow Transplant. 2005; 35: 85-89.

8. Ravandi F, O'Brien S, Thomas D, Faderl S, Jones D, Garris R, et al. First report of phase 2 study of dasatinib with hyper-CVAD for the frontline treatment of patients with Philadelphia chromosome-positive (Ph+) acute lymphoblastic leukemia. Blood. 2010; 116: 2070-2077.

9. Przepiorka D, Weisdorf D, Martin P, Klingemann HG, Beatty P, Hows J, et al. 1994 Consensus conference on acute GVHD grading. Bone Marrow Transplant. 1995; 15: 825-828.

10. Rashidi A, DeFor TE, Holtan SG, Blazar BR, Weisdorf DJ, MacMillan ML. Outcomes and predictors of response in steroid-refractory acute graft-versus-host disease. Biol Blood Marrow Transplant. 2019; 25: 2297-2302.

11. Perfetti P, Carlier P, Strada P, Gualandi F, Occhini D, Lint MT, et al., Extracorporeal photopheresis for the treatment of steroid refractory acute GVHD. Bone Marrow Transplant. 2008; 42: 609-617.

12. Feingold KR, Grunfeld C. Lipoprotein Apheresis. South Dartmouth: Endotext; 2020. Available from: https://www.ncbi.nlm.nih.gov/books/NBK425700.

13. Franklin M, Burns L, Perez S, Yerragolam D, Makenbaeva D. Incidence of type 2 diabetes mellitus and hyperlipidemia in patients prescribed dasatinib or nilotinib as first- or second-line therapy for chronic myelogenous leukemia in the US. Curr Med Res Opin. 2018; 34: 353-360.

14. Buhaescu I, Izzedine H. Mevalonate pathway: A review of clinical and therapeutical implications. Clin Biochem. 2007; 40: 575-584. 
15. Bellosta S, Paoletti R, Corsini A. Safety of statins: Focus on clinical pharmacokinetics and drug interactions. Circulation. 2004; 109: III50-III57.

16. Neuvonen PJ, Niemi M, Backman JT. Drug interactions with lipid-lowering drugs: Mechanisms and clinical relevance. Clin Pharmacol Ther. 2006; 80: 565-581.

17. Marini BL, Choi SW, Byersdorfer CA, Cronin S, Frame DG. Treatment of dyslipidemia in allogeneic hematopoietic stem cell transplant patients. Biol Blood Marrow Transplant. 2015; 21: 809-820.

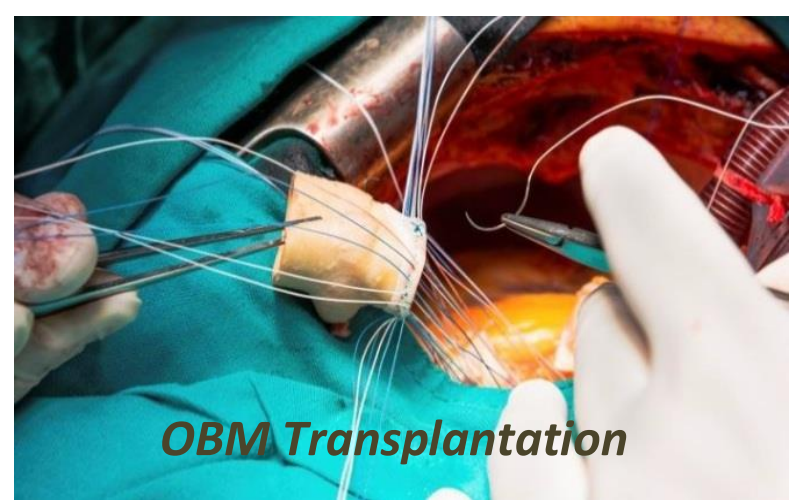

Enjoy OBM Transplantation by:

1. Submitting a manuscript

2. Joining in volunteer reviewer bank

3. Joining Editorial Board

4. Guest editing a special issue

For more details, please visit:

http://www.lidsen.com/journals/transplantation 